\title{
Coupled nonlinear Schrödinger equations with cubic-quintic nonlinearity: Integrability and soliton interaction in non-Kerr media
}

\author{
R. Radhakrishnan ${ }^{1}$, A. Kundu ${ }^{2}$ and M. Lakshmanan ${ }^{1}$ \\ ${ }^{1}$ Centre for Nonlinear Dynamics, Department of Physics, Bharathidasan University, Tiruchirapalli 620 024, India. \\ 2 Theory Group. Saha Institute of Nuclear Physics, 1/AF, Bidhan Nagar, Calcutta 700064 , India.
}

\begin{abstract}
We propose an integrable system of coupled nonlinear Schrödinger equations with cubic-quintic terms describing the effects of quintic nonlinearity on the ultra-short optical soliton pulse propagation in non-Kerr media. Lax pair, conserved quantities and exact soliton solutions for the proposed integrable model are given. Explicit form of two-solitons are used to study soliton interaction showing many intriguing features including inelastic (shape changing) scattering. Another novel system of coupled equations with fifth-degree nonlinearity is derived, which represents vector generalization of the known chiral-soliton bearing system.
\end{abstract}

\section{INTRODUCTION}

Optical solitons have promising potential to become principal carriers in telecommunication due to their capability of propagating long distances without attenuation and changing their shapes 1 - 1 . Therefore considerable attention is being paid theoretically and experimentally to analyse the dynamics of optical solitons in optical waveguides (for example silica fibers) under different contexts [1 6]. Such investigations are helpful for realizing optical soliton applications, particularly in soliton-based optical communication systems [5] and nonlinear optical switches [6]. The waveguides used in such optical systems are usually of Kerr type [7]. Consequently the dynamics of light pulses are described by the nonlinear Schrödinger (NLS) family of equations with cubic nonlinear terms [7,8]. However, as the intensity of the incident light field becomes stronger, non-Kerr nonlinearity effect comes into play and due to this additional effect, the physical features and the stability of NLS soliton can change [3].

The way through which non-Kerr nonlinearity influences NLS soliton propagation is described by the NLS family of equations with higher-degree nonlinear terms [9 16]. Therefore investigations on these evolution equations become important from a theoretical point of view. Particularly this importance has received a boost after the experimental observation of multistability of solitons in non-Kerr fiber [17]. In general the models proposed in the literature [3, 9 [16] for describing the non-Kerr effects are not completely integrable and can not be solved exactly by the inverse scattering transform method. In such non-integrable systems, therefore, the details of soliton-interaction during collision can not be described exactly and hence are still open to debate. However, numerical stimulations [18] show that even the slightest change from the Kerr nonlinearity results in the two solitons annihilating each other, merging or creating many new solitons, depending on the initial inclination of the two solitons and their shapes. But besides the important problem of computer time, the numerical approach is not very appealing in the sense that it is not a simple task to get physical insight from purely numerical experiments. The idea therefore is to use approximate analytical methods such as perturbation technique, variational method, etc., in order to compensate for the lack of exact results [19]. By treating the quintic nonlinear terms due to non-Kerr effect as perturbations of the cubic NLS equations, i.e. restricting the effects of quintic nonlinearity to be less predominant than the cubic terms, the NLS equations are studied both analytically and numerically in [3, 10, 13, 16, .

In this paper we have obtained for the first time (to our knowledge) an integrable system of coupled NLS equations including cubic-quintic terms describing the effects of quintic nonlinearity with arbitrary coupling, which generalizes the coupled hybrid NLS equations with cubic nonlinearity [20,21]. Lax pair as well as infinite set of conserved quantities are derived for the proposed integrable model. We also find exact soliton solutions for our model and using the explicit form of the two-soliton solution we study the associated soliton collision. A remarkable interrelation between the newly proposed integrable model and the celebrated Manakov model 22 helps to use the recently discovered general twosoliton solution of the later model [23]. This reveals the fascinating occurrence of shape changing inelastic soliton collision also in the present model, in addition to some other interesting features. We believe that such a study using higher-order solitons (multisolitons) becomes important in the light of the proposal by Hasegawa and Nyu [24] regarding "eigenvalue communication", in which the information may be transmitted by higher-order solitons and a recent one sugested by Jakubowski, Steiglitz and Squiev [25] on nontrivial information transmission system, which uses the more general two-soliton solution of the Manakov system [22] derived by Radhakrishnan, Lakshmanan and 
Hietarinta in [23]. We also find that the Hamiltonian of the present integrable model is associated with a non-canonical Poisson bracket (PB) structure. However, using the same Hamiltonian with canonical PB relations we derive another coupled system with quintic nonlinearirity, which may be transformed to a vector generalization of the chiral-solitonic model of Aglietti et al [33].

The plan of our paper is as follows. The basic evolution equation with cubic-quintic nonlinearity which describes the soliton evolution in non-Kerr media with parabolic nonlinearity is discussed in Sec-II. Sections III is devoted to the proposed model and presents its integrability property by explicit construction of the Lax pair as well as the hierarchy of conserved quantities generated through a recurrence relation, which in turn is derived from a coupled Ricatti equation using the Lax operator. Sec IV gives the exact soliton solution for our cubic-quintic nonlinear evolution equation and using the explicit analytic solution we study here the collision process of solitons so as to understand the influence of quintic nonlinear effects on the Manakov model. Section V establishes the interrelation and gauge transformation between our system and the Manakov model including the anyon-like nonultralocal PB for our model. Sec VI presents a vector generalization of the chiral soliton bearing system starting from the same Hamiltonian and discusses some of its interesting features. Sec VII gives concluding remarks.

\section{BASIC EVOLUTION EQUATIONS}

Generally when high optical intensities (or materials with high nonlinear coefficients even at moderate optical intensities, for example semiconductor doped glasses, organic polymers, thin liquid filled capillaries, etc.) are considered, it is necessary to take into account higher power nonlinearities arising from an expansion of the refractive index in powers of intensity $I$ of the light pulse : $n=n_{0}+n_{2} I+n_{4} I^{2}+\ldots$, where $n_{0}$ is the linear refractive index coefficient and $n_{2}, n_{4} \ldots$, are nonlinear refractive index coefficients [3, 10].

In the case of $n=n_{0}+n_{2} I+n_{4} I^{2}$, the wave equation for high-intensity light pulse propagation in an isotropic single-mode optical fiber with a circular cross-section and fiber axis z can be written as

$$
\nabla^{2} \mathbf{E}-\frac{1}{c^{2}} \frac{\partial^{2} \mathbf{D}_{\mathbf{L}}}{\partial t^{2}}=\frac{1}{c^{2}} \frac{\partial^{2} \mathbf{D}_{\mathbf{N L}}}{\partial t^{2}}
$$

where $c$ is the speed of light, the linear part $\mathbf{D}_{\mathbf{L}}$ and the nonlinear part $\mathbf{D}_{\mathbf{N L}}$ of the electric field displacements are related to the electric field $\mathbf{E}(\mathbf{r}, t)$ by the relation $\mathbf{D}_{\mathbf{L}}=\int_{0}^{\infty} \epsilon\left(t^{\prime}\right) \mathbf{E}\left(t-t^{\prime}\right) d t^{\prime}$ and $\mathbf{D}_{\mathbf{N L}}=\epsilon_{2}|E|^{2}+\epsilon_{4}|E|^{4}$, in which $\epsilon=n_{o}^{2}, \epsilon_{2}=2 n_{2} n_{0}$ and $\epsilon_{4}=2 n_{4} n_{0}$.

A solution of Eq.(1) is sought in the form

$$
\mathbf{E}=\mathbf{e} R(r) A(z, t) e^{i \beta z-i \omega t}
$$

where $\mathbf{e}$ is a unit vector in the direction of wave polarisation, $R(\mathbf{r})$ describes the transverse field modes, in which $\mathbf{r}$ is a two dimensional vector in the $\mathrm{x}-\mathrm{y}$ plane and $A(z, t)$ is a slowly varying amplitude. Here we assume that $R(\mathbf{r})$, which is mainly defined by the linear effects, corresponds to the modal distribution of the fundamental fiber mode $H E_{11}$, for simplicity. Then from the equations (1) and (2), assuming the temporal dispersion of the dielectric permitivity to be small, using the slowly varying envelope approximation and following the procedure in section 2.1 of 8 , the follwing nonlinear partial differential equation for $A(z, t)$ can be obtained,

$$
\begin{gathered}
i\left[A_{z}+\frac{1}{v_{g}} A_{t}\right]-\frac{1}{2} k_{\omega \omega} A_{t t}-\frac{i}{6} k_{\omega \omega \omega} A_{t t t}+\frac{k n_{2}}{n_{0}} \alpha_{0}|A|^{2} A \\
+\frac{k n_{4}}{n_{0}} \beta_{0}|A|^{4} A+\frac{i n_{2} \alpha_{0}}{v_{g} n_{0}}\left(|A|^{2} A\right)_{t}+\frac{i n_{4} \beta_{0}}{v_{g} n_{0}}\left(|A|^{4} A\right)_{t}=0,
\end{gathered}
$$

where the subscript $\omega$ of the wave number $k$ ( i.e. $k_{\omega}$ ) indicates differentiation of $\mathrm{k}$ with respect to $\omega$, the subscripts $t$ and $z$ of $A$ indicate differentiation of $A$ with respect to the coordinates $t$ and $z$ respectively and the numerical values of the parameters $\alpha_{0}$ and $\beta_{0}$ depend on the form of the function $R(\mathbf{r})$.

It is convenient now to transform the above equation to a reference frame moving with group velocity $v_{g}$, and to introduce dimensionless variables $q=\frac{A}{\mid A_{0}}, \gamma=\frac{2 n_{4} \beta_{0}\left|A_{0}\right|^{2}}{n_{2} \alpha_{0}}, \gamma_{1}=\frac{-k_{\omega \omega \omega}}{3\left(-k_{\omega \omega}\right)}\left[\frac{1}{z_{N L}\left(-k_{\omega \omega}\right)}\right]^{\frac{1}{2}}, \gamma_{2}=\frac{2}{v_{g}}\left[\frac{n_{2} \alpha_{0}\left|A_{0}\right|^{2}}{k n_{0}\left(-k_{\omega \omega}\right)}\right]^{\frac{1}{2}}$, $\frac{\gamma_{3}=2 n_{4} \beta_{0}\left|A_{0}\right|^{2}}{v_{g}\left[\frac{\left|A_{0}\right|^{2}}{k n_{0}{ }^{\alpha_{0}\left(-k_{\omega}\right)}}\right]^{\frac{1}{2}}}, z_{N L}^{-1}=\frac{k n_{2} \alpha_{0}\left|A_{0}\right|^{2}}{n_{0}}, t \rightarrow\left[\frac{1}{z_{N L}\left(-k_{\omega \omega}\right)}\right]^{\frac{1}{2}}\left(t-\frac{z}{v_{g}}\right)$ and $z \rightarrow \frac{z}{2 z_{N L}}$, in which $z_{N L}$ characterizes the 
nonlinear propeties of the fiber and $\left|A_{0}\right|$ is a measure of the maximum amplitude of the input pulse. Now equation (3) takes the form

$$
i q_{z}+q_{t t}+2|q|^{2} q+\gamma|q|^{4} q+i \gamma_{1} q_{t t t}+i \gamma_{2}\left(|q|^{2} q\right)_{t}+i \gamma_{3}\left(|q|^{4} q\right)_{t}=0 .
$$

Equation (4) describes the effects of quintic nonlinear terms proportional to the real parameters $\gamma$ and $\gamma_{3}$ on the dynamics of pulse envelope allowing self-phase modulation and higher-order linear and nonlinear dispersions. For pulse widths greater than $100 \mathrm{fs}$, one can neglect the last three terms of (4) and the resulting equation is a wellstudied [3,10] simple normalized NLS equation with cubic-quintic nonlinear terms. Further the system (4) is a special case of the dynamical equation considered for the fiber system with saturating nonlinearity [12].

It is of further interest to extend the above analysis to include multimode effects. For this purpose, there are several ways to generalize equation (4) to a set of coupled equations depending on the physical situations. A fairly general form of coupled nonlinear Schrödinger (CNLS) equation with cubic-quintic nonlinearity is

$$
\begin{aligned}
i q_{1 z}+q_{1 t t}+ & 2\left(\left|q_{1}\right|^{2}+B\left|q_{2}\right|^{2}\right) q_{1}+\gamma\left(\left|q_{1}\right|^{2}+B\left|q_{2}\right|^{2}\right)^{2} q_{1}+\rho q_{1}+\kappa q_{2} \\
- & i \mu\left[\gamma_{1} q_{1 t t t}+\gamma_{2}\left[\left(\left|q_{1}\right|^{2}+B\left|q_{2}\right|^{2}\right) q_{1 t}+\left(q_{1} q_{1 t}^{*}+B q_{2} q_{2 t}^{*}\right) q_{1}\right.\right. \\
& \left.\left.+\gamma^{\prime}\left(q_{1 t} q_{1}^{*}+B q_{2 t} q_{2}^{*}\right) q_{1}\right]+\gamma_{3}\left[\left(\left|q_{1}\right|^{2}+B\left|q_{2}\right|^{2}\right)^{2} q_{1}\right] t\right]=0 . \\
i q_{2 z}+q_{2 t t}+ & 2\left(B\left|q_{1}\right|^{2}+\left|q_{2}\right|^{2}\right) q_{2}+\gamma\left(B\left|q_{1}\right|^{2}+\left|q_{2}\right|^{2}\right)^{2} q_{2}-\rho q_{2}+\kappa q_{1} \\
- & i \mu\left[\gamma_{1} q_{2 t t t}+\gamma_{2}\left[\left(B\left|q_{1}\right|^{2}+\left|q_{2}\right|^{2}\right) q_{2 t}+\left(B q_{1} q_{1 t}^{*}+q_{2} q_{2 t}^{*}\right) q_{2}\right.\right. \\
& \left.\left.+\gamma^{\prime}\left(B q_{1 t} q_{1}^{*}+q_{2 t} q_{2}^{*}\right) q_{2}\right]+\gamma_{3}\left[\left(B\left|q_{1}\right|^{2}+\left|q_{2}\right|^{2}\right)^{2} q_{2}\right]_{t}\right]=0 .
\end{aligned}
$$

A nonlinear direction coupler with quintic nonlinearity(or parabolic nonlinearity coupler) has $B=\rho=\mu=0$ [26]. For $\mu=\gamma=0$, Eq.(5) acts as a mathematical model for a periodically twisted elliptical birefringent fiber [27]. If $\gamma=\rho=\kappa=\gamma_{1}=\gamma_{3}=0$, and $\gamma^{\prime}=1$ then Eq.(3) becomes the coupled hybrid nonlinear Schrödinger equation [20] used to investigate the effects of birefringence on pulse propagation in the femto-second range. In the absence of quintic nonlinear terms proportional to the real parameters $\gamma$ and $\gamma_{3}$, soliton interaction supported by system (5) has been studied by deriving higher-order soliton solutions under the parameteric restrictions $B=1$ and $3 \gamma_{1}=\gamma_{2}$ [28]. One may also note that when $B=1$ and $\gamma^{\prime}=1$ the linear coupling terms proportional to the parameter $\rho$ and $\kappa$ in Eq.(5) can be removed without affecting the other terms by using the transformation

$$
\begin{aligned}
& q_{1} \rightarrow \cos \left(\frac{\theta}{2}\right) e^{i \Gamma z} q_{1}-\sin \left(\frac{\theta}{2}\right) e^{-i \Gamma z} q_{2}, \\
& q_{2} \rightarrow \sin \left(\frac{\theta}{2}\right) e^{i \Gamma z} q_{1}+\cos \left(\frac{\theta}{2}\right) e^{-i \Gamma z} q_{2},
\end{aligned}
$$

where $\Gamma=\left(\rho^{2}+\kappa^{2}\right)^{\frac{1}{2}}$ and $\theta=\tan ^{-1}\left(\frac{\kappa}{\rho}\right)$. If the nonlinearity is restricted only to cubic terms corresponding to pulse widths greater than 100fs, one obtains the celebrated integrable Manakov model [22, 35]

$$
\begin{aligned}
& i q_{1 M z}+q_{1 M t t}+2\left(\left|q_{1 M}\right|^{2}+\left|q_{2 M}\right|^{2}\right) q_{1 M}=0, \\
& i q_{2 M z}+q_{2 M t t}+2\left(\left|q_{1 M}\right|^{2}+\left|q_{2 M}\right|^{2}\right) q_{2 M}=0 .
\end{aligned}
$$

There is a large amount of theoretical work [1] [5] devoted to the CNLS family of equations with cubic nonlinearity. However, to the knowledge of the authors, to date the CNLS equations with non-Kerr nonlinearity have received very little attention in the literature, particularly in connection with the integrability aspects. In the following sections, by identifying one such integrable nonlinear evolution equation, we derive the two-soliton solution so as to get some idea about soliton interaction in non-Kerr media.

\section{INTEGRABILITY PROPERTY OF THE PROPOSED MODEL: LAX PAIR AND CONSERVED QUANTITIES}

It is evident that eq. (5) does not exhibit the explicit rotational symmetry in the internal space spanned by the vector $\left(q_{1}, q_{2}\right)$. However, for $B=1$ such a symmetry is restored. Assuming further that $\gamma_{1}=\gamma_{3}=\gamma^{\prime}=0$, (5) can be reduced to the following quintic generalization of the coupled cubic NLS equation, 


$$
\begin{array}{r}
i q_{1 z}+q_{1 t t}+2\left(\left|q_{1}\right|^{2}+\left|q_{2}\right|^{2}\right) q_{1}+\gamma\left(\left|q_{1}\right|^{2}+\left|q_{2}\right|^{2}\right)^{2} q_{1}+\rho q_{1}+\kappa q_{2}-i \mu \gamma_{2} \\
{\left[\left(\left|q_{1}\right|^{2}+\left|q_{2}\right|^{2}\right) q_{1 t}+\left(q_{1} q_{1 t}^{*}+q_{2} q_{2 t}^{*}\right) q_{1}\right]=0,} \\
i q_{2 z}+q_{2 t t}+2\left(\left|q_{1}\right|^{2}+\left|q_{2}\right|^{2}\right) q_{1}+\gamma\left(\left|q_{1}\right|^{2}+\left|q_{2}\right|^{2}\right)^{2} q_{2}-\rho q_{2}+\kappa q_{1}-i \mu \gamma_{2} \\
{\left[\left(\left|q_{1}\right|^{2}+\left|q_{2}\right|^{2}\right) q_{2 t}+\left(q_{1} q_{1 t}^{*}+q_{2} q_{2 t}^{*}\right) q_{2}\right]=0 .}
\end{array}
$$

The terms $\rho$ and $\kappa$ can be removed from (8) by using transformation (6). Eq. (8) without quintic nonlinearity was investigated in [21]. However the remarkable fact is that (8) itself can be shown to be exactly integrable. Our proposed model is a further generalization of (8) and naturally of (7), where the internal rotational symmetry is broken again and more parameters are introduced with arbitrary values, which can be chosen conveniently to suit the real situations. The model can be given as

$$
\begin{array}{r}
i q_{1 z}+q_{1 t t}+2\left(\left|q_{1}\right|^{2}+\left|q_{2}\right|^{2}\right) q_{1}+\left(\rho_{1}\left|q_{1}\right|^{2}+\rho_{2}\left|q_{2}\right|^{2}\right)^{2} q_{1}+2 \rho_{2}\left[\left(\tau_{1}-\rho_{1}\right)\left|q_{1}\right|^{2}+\left(\tau_{2}-\rho_{2}\right)\right. \\
\left.\left|q_{2}\right|^{2}\right]\left|q_{2}\right|^{2} q_{1}-2 i\left[\left(\rho_{1}\left|q_{1}\right|^{2}+\rho_{2}\left|q_{2}\right|^{2}\right) q_{1}\right]_{t}+2 i\left(\rho_{1} q_{1}^{*} q_{1 t}+\rho_{2} q_{2}^{*} q_{2 t}\right) q_{1}=0, \\
i q_{2 z}+q_{2 t t}+2\left(\left|q_{1}\right|^{2}+\left|q_{2}\right|^{2}\right) q_{2}+\left(\tau_{1}\left|q_{1}\right|^{2}+\tau_{2}\left|q_{2}\right|^{2}\right)^{2} q_{2}+2 \tau_{1}\left[\left(\rho_{1}-\tau_{1}\right)\left|q_{1}\right|^{2}+\left(\rho_{2}-\tau_{2}\right)\right. \\
\left.\left|q_{2}\right|^{2}\right]\left|q_{1}\right|^{2} q_{2}-2 i\left[\left(\tau_{1}\left|q_{1}\right|^{2}+\tau_{2}\left|q_{2}\right|^{2}\right) q_{2}\right]_{t}+2 i\left(\tau_{1} q_{1}^{*} q_{1 t}+\tau_{2} q_{2}^{*} q_{2 t}\right) q_{2}=0 .
\end{array}
$$

where $\rho_{1}, \rho_{2}, \tau_{1}$ and $\tau_{2}$ are real free parameters. It is evident that with a symmetric reduction $\rho_{1}=\rho_{2}=\tau_{1}=\tau_{2}$, we can recover (8) from (9), while a different reduction with $q_{1}=q$ and $q_{2}=0$ (or $q_{1}=0$ and $q_{2}=q$ ) yields the integrable Kundu-Eckhaus equation [29]

$$
i q_{z}+q_{t t}+2|q|^{2} q+\rho_{1}^{2}|q|^{4} q-2 i \rho_{1}\left(|q|^{2}\right)_{t} q=0 .
$$

Importantly this generalized model (9) turns out also to be exactly integrable. For establishing the integrability property of the proposed system, which consequently proves also the integrability of the reduced model (8), we find the Lax pair $(L, M)$ associated with (9) as

$$
\begin{array}{r}
L=\left(\begin{array}{ccc}
-i \lambda & q_{1} & q_{2} \\
-q_{1}^{*} & -i \theta_{1 t}+i \lambda & 0 \\
-q_{2}^{*} & 0 & -i \theta_{2 t}+i \lambda
\end{array}\right), \\
M=\left(\begin{array}{ccc}
{\left[-2 i \lambda^{2}+i\left(\left|q_{1}\right|^{2}+\left|q_{2}\right|^{2}\right)\right],} & 2 \lambda q_{1}+i q_{1 t}+\theta_{1 t} q_{1}, & 2 \lambda q_{2}+i q_{2 t}+\theta_{2 t} q_{2} \\
-2 \lambda q_{1}^{*}+i q_{1 t}^{*}-\theta_{1 t} q_{1}^{*}, & {\left[2 i \lambda^{2}-i\left|q_{1}\right|^{2}-i \theta_{1 z}\right],} & -i q_{1}^{*} q_{2} \\
-2 \lambda q_{2}^{*}+i q_{2 t}^{*}-\theta_{2 t} q_{2}^{*}, & -i q_{1} q_{2}^{*}, & {\left[2 i \lambda^{2}-i\left|q_{2}\right|^{2}-i \theta_{2 z}\right]}
\end{array}\right),
\end{array}
$$

where

$$
\theta_{1}=\int_{-\infty}^{t}\left(\rho_{1}\left|q_{1}\right|^{2}+\rho_{2}\left|q_{2}\right|^{2}\right) d t^{\prime}, \quad \theta_{2}=\int_{-\infty}^{t}\left(\tau_{1}\left|q_{1}\right|^{2}+\tau_{2}\left|q_{2}\right|^{2}\right) d t^{\prime}
$$

Here $\lambda$ is the spectral parameter. It may be easily checked that the zero curvature condition $L_{z}-M_{t}+[L, M]=0$, with the explicit Lax operators (11), yields Eq. (9). In section $\mathrm{V}$ we give another evidence of its integrability by relating system (9) to the integrable Manakov model through a gauge transformation of the pair (11) to the Manakov Lax operators.

Integrable systems, as is well known, possess infinite number of conserved quantities in involution, of which usually the lower ones are of physical importance. Explicit forms of such conserved quantities for the integrable system (9) can be derived from a recurrence relation obtained from the Ricatti equation related to the Lax operator. For this purpose we use the linear system related to (11a)

$$
\Phi_{t}(\lambda, t)=L(\lambda, t) \Phi(\lambda, t), \Phi=\left(\phi_{1}, \phi_{2}, \phi_{3}\right)
$$

and observe that

$$
\ln a(\lambda)=\left.\ln \phi_{1} e^{-i \lambda t}\right|_{t \rightarrow \infty}=\sum_{n} c_{n} \lambda^{-n}
$$

serves as the generator of the conserved quantities $\left\{c_{n}\right\}$ through an expansion in the spectral parameter $\lambda$. The first equation of the system (12) thus yields the relation 


$$
c_{n}=\int_{-\infty}^{+\infty} d t\left(q_{1} \Gamma_{n}^{(1)}+q_{2} \Gamma_{n}^{(2)}\right), \quad n \geq 1
$$

with the expansion $\Gamma^{(a)}=\sum_{n=1}^{\infty} \Gamma_{n}^{(a)} \lambda^{-n}, a=1,2$, where we have denoted $\Gamma^{(1)}=\frac{\phi_{2}}{\phi_{1}}$ and $\Gamma^{(2)}=\frac{\phi_{3}}{\phi_{1}}$. For finding now the infinite set of conserved quantities we may use the rest of the equations of $(12)$ to derive a set of two coupled Ricatti equations for $\Gamma^{(1)}(\lambda)$ and $\Gamma^{(2)}(\lambda)$. Expanding in powers of $\lambda$ as mentioned above we obtain the recurrent relations

$$
\begin{aligned}
& -2 i \Gamma_{n+1}^{(1)}=\Gamma_{n t}^{(1)}+i \theta_{1 t} \Gamma_{n}^{(1)}+q_{1} \sum_{i=1}^{n-1} \Gamma_{n-i}^{(1)} \Gamma_{i}^{(1)}+q_{2} \sum_{i=1}^{n-1} \Gamma_{n-i}^{(1)} \Gamma_{i}^{(2)}, \\
& -2 i \Gamma_{n+1}^{(2)}=\Gamma_{n t}^{(2)}+i \theta_{2 t} \Gamma_{n}^{(2)}+q_{2} \sum_{i=1}^{n-1} \Gamma_{n-i}^{(2)} \Gamma_{i}^{(2)}+q_{1} \sum_{i=1}^{n-1} \Gamma_{n-i}^{(2)} \Gamma_{i}^{(1)},
\end{aligned}
$$

with $\Gamma_{1}^{(a)}=-\frac{i}{2} q_{a}^{*}$. This gives finally the conserved quantities in the explicit form as

$$
\begin{aligned}
c_{1} & =-\frac{1}{2 i} \int_{-\infty}^{+\infty} d t\left(\left|q_{1}\right|^{2}+\left|q_{2}\right|^{2}\right) \\
c_{2} & =-\frac{i}{4} \int_{-\infty}^{+\infty} d t\left[-i\left(q_{1} q_{1 t}^{*}+q_{2} q_{2 t}^{*}\right)+\rho_{1}\left|q_{1}\right|^{4}+\tau_{2}\left|q_{2}\right|^{4}+\left(\rho_{2}+\tau_{1}\right)\left|q_{1}\right|^{2}\left|q_{2}\right|^{2}\right] \\
c_{3} & =-\frac{i}{8} \int_{-\infty}^{+\infty} d t\left[\left(q_{1} q_{1 t t}^{*}+q_{2} q_{2 t t}^{*}\right)+\left(\left|q_{1}\right|^{2}+\left|q_{2}\right|^{2}\right)^{2}+\right. \\
& \left.i\left(\left|q_{1}\right|^{2} N_{1 t}+\left|q_{2}\right|^{2} N_{2 t}\right)+2 i\left(N_{1} q_{1} q_{1 t}^{*}+N_{2} q_{2} q_{2 t}^{*}\right)-\left(N_{1}^{2}\left|q_{1}\right|^{2}+N_{2}^{2}\left|q_{2}\right|^{2}\right)\right],
\end{aligned}
$$

etc., where $N_{1}=\theta_{1 t}=\rho_{1}\left|q_{1}\right|^{2}+\rho_{2}\left|q_{2}\right|^{2}$ and $N_{2}=\theta_{2 t}=\tau_{1}\left|q_{1}\right|^{2}+\tau_{2}\left|q_{2}^{2}\right|$. The above conserved quantities in (15, 17 ) may be interpreted in terms of the number operator $N$, the total momentum $P$ and the total energy or the Hamiltonian of the system $H$. However it is intriguing to remark that since here the fields $q_{a}$, $q_{a}^{*}$ do not have canonical Poisson bracket relations some care has to be taken in deriving the equation of motion (9) from the Hamiltonian (17) . In fact the Poisson bracket structures of the fields, which are derived in sec V, show an interesting anyon-like feature.

\section{EXACT SOLITON SOLUTIONS AND SCATTERING OF SOLITONS IN THE GENERALIZED MANAKOV MODEL IN NON-KERR MEDIA}

The proposed system (9) with quintic nonlinearity allows exact $N$-soliton solutions, which can be found for example by Hirota's method following the same procedure as in the Manakov model. However a more direct and convenient way is to use the known solutions of the Manakov model (7) themselves for constructing the soliton solutions of (9). This is possible due to the interrelation between these two models, which will be established in the next section. Thus we find the explicit 1-soliton solution of (9) in the form

$$
\left(q_{1}, q_{2}\right)=\left(\alpha e^{i \delta_{1} \tanh (\nu(t-v z+\delta))}, \beta e^{i \delta_{2} \tanh (\nu(t-v z+\delta))}\right) \operatorname{sech}(\nu(t-v z+\delta)) e^{i(\kappa t+\omega z)},
$$

where different parameters of the solution are related with the spectral parameter $\lambda=\nu+i \kappa$ and the parameters of the model as

$$
v=2 \kappa, \quad \omega=\nu^{2}-\kappa^{2}, \quad \delta_{1}=\frac{1}{\nu}\left(\rho_{1}|\alpha|^{2}+\rho_{2}|\beta|^{2}\right), \quad \delta_{2}=\frac{1}{\nu}\left(\tau_{1}|\alpha|^{2}+\tau_{2}|\beta|^{2}\right)
$$

together with a constant phase $\delta$. Comparing with the Manakov soliton we see that there is an interesting phase change in the carrier wave. The plane wave like character in the Manakov model has been deformed into a wave suffering compression and rarification of the phases in a kink-like profile (see Fig. 1a,b). This shows that the effect of quintic nonlinearity of our model appears in the soliton phases and therefore in the derivatives of the soliton profile $q_{a x}, q_{a t}$, which must also change the momentum and energy of the soliton.

Now exploiting the higher-soliton solutions of the Manakov model it is also possible to find higher-solitons for (9) in explicit form. The Manakov model (77) has received considerable attention in recent years [35, 36, 23, in order to understand the soliton collision in birerefringent fiber. However the importance of finding higher-soliton solutions in 
explicit form has been understood only quite recently [23,25]. By constructing the most general two-soliton solution of the integrable Manakov model, two of the authors (R.R and M.L) and Hietarinta [23] have shown that the soliton in birefringent fiber can in general change its shape after interaction due to an intensity redistribution among the modes, eventhough the total intensity remains conserved. This shape changing collision arising essentially due to the change in polarisation angle helps to realise the exciting possibility of switching between components. (However, the standard shape-preserving collision property of the $(1+1)$ dimensional soliton system is recoverd, when restrictions are imposed on some of the free parameters in the two-soliton solution [23] ). Recently using the shape changing collision concept, Jakubowski, Steiglitz and Squier 25] have designed sequences of solitons operating on other sequences of solitons that effect logic operations and suggested nontrivial information transformation system.

Now in order to investigate the implication of this property of soliton when the additional cubic and quintic nonlinear terms are included, we construct the two-soliton solution of the system (9) and expect again a non-trivial change in the soliton phase as in the case of 1-soliton solution. Such a two-soliton solution expressed compactly through that of the Manakov system (7) $\left(q_{1 M}, q_{2 M}\right)$ takes the form (see eq.(24) below)

$$
q_{1}=q_{1 M} e^{i \int\left(\rho_{1}\left|q_{1 M}\right|^{2}+\rho_{2}\left|q_{2 M}\right|^{2}\right) d t}, \quad q_{2}=q_{2 M} e^{i \int\left(\tau_{1}\left|q_{1 M}\right|^{2}+\tau_{2}\left|q_{2 M}\right|^{2}\right) d t},
$$

where a general two-soliton solution [23] of the Manakov model is given by

$$
\left(q_{1 M}, q_{2 M}\right)=\frac{\left(\alpha_{1}, \beta_{1}\right) e^{\eta_{1}}+\left(\alpha_{2}, \beta_{2}\right) e^{\eta_{2}}+\left(e^{\delta_{1}}, e^{\delta_{1}^{\prime}}\right) e^{\eta_{1}+\eta_{1}{ }^{*}+\eta_{2}}+\left(e^{\delta_{2}}, e^{\delta_{2}^{\prime}}\right) e^{\eta_{1}+\eta_{2}+\eta_{2}{ }^{*}}}{1+e^{\eta_{1}+\eta_{1}{ }^{*}+R_{1}}+e^{\eta_{1}+\eta_{2}{ }^{*}+\delta_{0}}+e^{\eta_{1}{ }^{*}+\eta_{2}+\delta_{0}{ }^{*}}+e^{\eta_{2}+\eta_{2}{ }^{*}+R_{2}}+e^{\eta_{1}+\eta_{1}{ }^{*}+\eta_{2}+\eta_{2}{ }^{*}+R_{3}}},
$$

in which $\eta_{j}=k_{j}\left(t+i k_{j} z\right), j=1,2, e^{\delta_{0}}=\frac{\kappa_{12}}{k_{1}+k_{2}^{*}}, e^{R_{1}}=\frac{\kappa_{11}}{k_{1}+k_{1}^{*}}, e^{R_{2}}=\frac{\kappa_{22}}{k_{2}+k_{2}^{*}}, e^{\delta_{1}}=\frac{k_{1}-k_{2}}{\left(k_{1}+k_{1}^{*}\right)\left(k_{1}^{*}+k_{2}\right)}\left(\alpha_{1} \kappa_{21}-\alpha_{2} \kappa_{11}\right)$, $e^{\delta_{2}}=\frac{k_{2}-k_{1}}{\left(k_{2}+k_{2}^{*}\right)\left(k_{1}+k_{2}^{*}\right)}\left(\alpha_{2} \kappa_{12}-\alpha_{1} \kappa_{22}\right), e^{\delta_{1}^{\prime}}=\frac{k_{1}-k_{2}}{\left(k_{1}+k_{1}^{*}\right)\left(k_{1}^{*}+k_{2}\right)}\left(\beta_{1} \kappa_{21}-\beta_{2} \kappa_{11}\right), e^{\delta_{2}^{\prime}}=\frac{k_{2}-k_{1}}{\left(k_{2}+k_{2}^{*}\right)\left(k_{1}+k_{2}^{*}\right)}\left(\beta_{2} \kappa_{12}-\beta_{1} \kappa_{22}\right), e^{R_{3}}=$ $\frac{\left|k_{1}-k_{2}\right|^{2}}{\left(k_{1}+k_{1}^{*}\right)\left(k_{2}+k_{2}^{*}\right)\left|k_{1}+k_{2}^{*}\right|^{2}}\left(\kappa_{11} \kappa_{22}-\kappa_{12} \kappa_{21}\right)$ and $\kappa_{i j}=\frac{\left(\alpha_{i} \alpha_{j}{ }^{*}+\beta_{i} \beta_{j}{ }^{*}\right)}{k_{i}+k_{j}}$. The six arbitrary complex parameters $\alpha_{1}, \alpha_{2}, \beta_{1}, \beta_{2}, k_{1}$ and $k_{2}$ determine the amplitude, velocity and phase of the asymptotic soliton. As we have detected already, we see also here that the two-soliton solution (19) of (9) differs from that of the Manakov model in phase-terms in a nontrivial way. Clearly, the phase change depends on the values of the real free parameters $\rho_{1}, \rho_{2}, \tau_{1}$ and $\tau_{2}$, and vanishes for the trivial choice giving back the Manakov soliton. A natural question therefore arises: Does this change in phase of $\left(q_{1}, q_{2}\right)$ for non-zero values of $\rho_{1}, \rho_{2}, \tau_{1}$ and $\tau_{2}$, which in turn accounts for the effect of the quintic terms in (9), make any qualitative change in the behaviour of soliton collision? This can be directly studied using the two-soliton solution (19-20) parallel to the procedure of the Manakov model given in Ref. [23]. In order to answer the above question, we have plotted pictures of soliton collision corresponding to the function $\left(\left|q_{1 t}\right|^{2},\left|q_{2 t}\right|^{2}\right)$ instead of $\left(\left|q_{1}\right|^{2},\left|q_{2}\right|^{2}\right)$, since due to $\left(\left|q_{1}\right|^{2},\left|q_{2}\right|^{2}\right)=\left(\left|q_{1 M}\right|^{2},\left|q_{2 M}\right|^{2}\right)$ one has to look into the derivatives of the fields, where the effect of phase terms are reflected. For comparing with the pure Manakov model let us consider first the case $\rho_{1}=\rho_{2}=\tau_{1}=\tau_{2}=0$. Fig. 2, shows the asymptotic forms of $\left(\left|q_{1 t}\right|^{2},\left|q_{2 t}\right|^{2}\right)$ for this case at $z=\mp 7$ for the parameter values $k_{1}=1.5+i 0.5, k_{2}=2.0-i 0.7, \alpha_{1}=\beta_{1}=\beta_{2}=1$, and $\alpha_{2}=\frac{(39+i 80)}{89}$. At $z=-7$, we have two well seperated asymptotic profiles as shown in Fig. 2a. During the propagation, these two solitary profiles interact with each other as shown in Fig. 3 and change form after interaction. For example, at $z=+7$ they have the profiles as shown in Fig. 2b. The change in shape disappears if we apply the elastic collision (shape-preserving collision) condition, namely $\alpha_{1}: \alpha_{2}=\beta_{1}: \beta_{2}$ by following the work of [23]. In Figs. 2-3, as mentioned, there is a splitting in each of the asymptotic profiles which appear before and after interaction. This happens due to the following reason. In the case corresponding to the Manakov model one obtains

$$
\left.q_{j M t}^{n \mp} \sim A_{j}^{n \mp} k_{n R} e^{i \eta_{n I}} \operatorname{sech}\left(\eta_{n R}+\phi^{n \mp}\right)\left\{\left[-k_{n R} \tanh \left(\eta_{n R}+\phi^{n \mp}\right)\right]+i k_{n I}\right\}\right|_{z \rightarrow \pm \infty}, j, n=1,2
$$

where $\eta_{n R}=k_{n R}\left(t-2 k_{n I} z\right), \eta_{n I}=k_{n I} t+\left(k_{n R}^{2}-k_{n I}^{2}\right) z$, the subscript $\mathrm{j}$ denotes mode while superscript $n \mp$ is used to define the two different interacting solitary waves appearing at $z \sim \mp \infty$ and $A_{j}^{n \mp}$ and $\phi^{n \mp}$ determine unit polarization vector and phase of the modes as defined in Ref. 23. From (21) one can note that for suitable choice of the parameters $k_{n R}$ and $k_{n I}$, the solitary waves get peaked around two values as shown in Figs. 2-3.

Now to investigate the effect of non-zero values of $\rho_{1}, \rho_{2}, \tau_{1}$ and $\tau_{2}$ or in other words to see the nontrivial contributions due to cubic-quintic generalization (9), we evaluate $q_{j t}=\left[q_{j M t}+i q_{j M} \theta_{j t}\right] \exp \left(i \theta_{j}\right), \quad j=1,2$. and plot the asymptotic behavior of the two-soliton solution (19) to the quintic generalization of the Manakov model Eq.(9) in Figs. 4a,b. The corresponding interaction profile of the solitons during their propagation is shown in Fig. 5. We observe firstly that, as in the case of Manakov model, here also generically the fascinating shape changing inelastic collision persists. However, in this case one can overcome the splitting effect of Figs. 2-3 corresponding to the Manakov 
model. For example, if we set $\rho_{1}=\rho_{2}=\tau_{1}=\tau_{2}=1$, in (19) then the splitting of solitons disappear, as evident from Figs. 4-5. The reason for this is that now we have

$$
\left|q_{j t}\right|^{2}=\left|q_{j M t}\right|^{2}+\left|q_{j M}\right|^{2}\left|\theta_{j t}\right|^{2}=\left|q_{j M t}\right|^{2}+\left|q_{j M}\right|^{2}\left(\delta_{1}\left|q_{1 M}\right|^{2}+\delta_{2}\left|q_{2 M}\right|^{2}\right),
$$

where $\delta_{1}$ and $\delta_{2}$ are equal to $\rho_{1}$ and $\rho_{2}$ for $j=1$, while to $\tau_{1}$ and $\tau_{2}$ for $j=2$. Since here the second term dominates over the first in the region of splitting, the splitting effect naturally gets supressed. Comparing Figs. 3 and 5 it is also important to note that the intensity of solitons $\left(\left|q_{1 t}\right|^{2},\left|q_{2 t}\right|^{2}\right)$ at the intersection region for solution (19) of our generalized model is much higher than that for (20) corresponding to the Manakov model. The above processes vividly demonstrate the nontrivial effect of the additional terms involving parameters $\rho_{1}, \rho_{2}, \tau_{1}$ and $\tau_{2}$ appearing in Eq.(9).

\section{RELATION WITH THE MANAKOV MODEL}

As we have mentioned above there exists an interesting interrelation between the quintic generalization (9) and the Manakov model (7), which in fact we have used already in deriving the soliton solutions of (9). We establish now this relationship by showing that the Lax operators of these two models are related through a local gauge transformation 29 31], while the fields are connected by a nonlinear transformation in dependent variables.

It is known [32] that under a gauge transformation of the Jost function $\Phi^{\prime}=g \Phi$ with the gauge field $g \in U(3)$, the Lax operators transform as

$$
L^{\prime}=g^{-1} L g-g^{-1} g_{t}, \quad M^{\prime}=g^{-1} M g-g^{-1} g_{z} .
$$

Choosing now the specific form

$$
g=\left(\begin{array}{ccc}
1 & 0 & 0 \\
0 & \exp \left(-i \theta_{1}\right) & 0 \\
0 & 0 & \exp \left(-i \theta_{2}\right)
\end{array}\right)
$$

with its elements $\theta_{1}, \theta_{2}$ being the same functions of $\mathrm{z}$ and $\mathrm{t}$ as in Eq. (11c) and performing the transformation (22), one can conveniently remove the diagonal terms involving $\theta_{1 t}, \theta_{2 t}$ and $\theta_{1 z}, \theta_{2 z}$ in the Lax pair (11a,b). It can be observed further that the resultant gauge transformed Lax operators reduce exactly to those of the Manakov model 22.35] if we introduce transformed fields

$$
q_{a M}=q_{a} \exp \left(-i \theta_{a}\right), \quad a=1,2
$$

along with their conjugates. At the same time transformation (24) reduces the equations (9) to those of the Manakov model (7).

The above points establish the relationship between these models and justifies the form of soliton solution presented in the earlier section for the model (9). Moreover, this procedure also provides an alternative proof of the integrability of our model. It is important to note that under such gauge transformation the Poisson bracket structure of the fields also gets changed. To find such changes in the canonical structure we may use transformation (24) to express our field through the Manakov fields and assuming standard canonical relation (26) for the Manakov model, we can derive the anyon-like relations for the fields of (9):

$$
\begin{aligned}
& \left\{q_{1}(x), q_{1}^{*}(y)\right\}=\delta(x-y)+i \rho_{1} \epsilon(x-y) q_{1}(x) q_{1}^{*}(y), \\
& \left\{q_{1}(x), q_{1}(y)\right\}=i \rho_{1} \epsilon(y-x) q_{1}(x) q_{1}(y), \\
& \left\{q_{1}(x), q_{2}(y)\right\}=-i\left(\rho_{2} \theta(x-y)-\tau_{1} \theta(y-x)\right) q_{1}(x) q_{2}(y), \\
& \left\{q_{1}(x), q_{2}^{*}(y)\right\}=i\left(\rho_{2} \theta(x-y)-\tau_{1} \theta(y-x)\right) q_{1}(x) q_{2}^{*}(y),
\end{aligned}
$$

etc., where $\epsilon(x)=\theta(x)-\theta(-x)$ is the sign function defined through the step function: $\theta(x)=1$ for $x>0, \theta(x)=0$, for $x \leq 0$. Note that at $x=y$ the fields exhibit canonical property, while at $x \neq y$ their behavior is nonultralocal and mimics anyon-like properties 34 in the classical limit. It may be remarked here that the generalized Manakov equation (9) can be derived directly from the Hamiltonian (17) by careful application of the PB structure (25) and the relation $\partial_{x} \theta(x-y)=\delta(x-y)$. 


\section{VECTOR GENERALIZATION OF CHIRAL SOLITONIC MODEL}

We have seen that for obtaining (9) from the Hamiltonian (17) we have to use noncanonical brackets (25). On the other hand if nevertheless one considers them to be canonical, i.e.

$$
\left\{q_{i}(x), q_{j}^{*}(y)\right\}=\delta(x-y) \delta_{i j},\left\{q_{i}(x), q_{j}(y)\right\}=0,
$$

from the same Hamiltonian (17) we can derive completely different coupled equations with fifth-degree nonlinearity. If for simplicity we assume $\rho_{1}=\rho_{2}=\tau_{1}=\tau_{2}=\rho_{0}$, we can derive these equations easily from $c_{3}$ as

$$
i q_{1 z}+q_{1 t t}+2\left(\left|q_{1}\right|^{2}+\left|q_{2}\right|^{2}\right) q_{1}-3 \rho_{0}^{2}\left(\left|q_{1}\right|^{2}+\left|q_{2}\right|^{2}\right)^{2} q_{1}-2 i \rho_{0}\left(\left(\left|q_{1}\right|^{2}+\left|q_{2}\right|^{2}\right) q_{1 t}+\left(q_{1}^{*} q_{1 t}+q_{2}^{*} q_{2 t}\right) q_{1}\right)=0
$$

and similarly for $q_{2}$ by interchanging the indices $1 \leftrightarrow 2$ in (27). We notice that this system of coupled equations again with cubic-quintic nonlinearity is a new system which is different from Eq. (8) presented earlier. To anlyse these equations more closely we perform again a nonlinear variable change as $q_{a} \rightarrow Q_{a}=q_{a} e^{-i \rho_{0} \theta}$ with $\theta_{t}=N \equiv$ $\left|Q_{1}\right|^{2}+\left|Q_{2}\right|^{2}$. After some lenghty but simple manipulations one can reduce the system (27) further to a more compact form with only cubic nonlinearity:

$$
i Q_{a z}+Q_{a t t}+2\left(N-\rho_{0} j\right) Q_{a}=0
$$

where we have denoted $j=j_{1}+j_{2}, j_{a}=i\left(Q_{a}^{*} Q_{a t}-Q_{a} Q_{a t}^{*}\right)$. We immediately recognize that this is nothing but the vector generalization of the Aglietti et al equation [33], however with the addition of a cubic nonlinearity coming from the Manakov model. Nevertheless the system (28) shows remarkable property close to the chiral-soliton feature of [33. In particular assuming the 1 -soliton form as $Q_{a}=A_{a} s(t-v z) e^{i\left(\frac{v}{2} t+\omega z\right)}$ one may conclude that here the quantity $\kappa=1+v \rho_{0}$ acts as the effective coupling constant of the nonlinear term, which regulates the intensity of the soliton. Therefore for the soliton velocity $v>-\frac{1}{\rho_{0}}$ only (with $\rho_{0}>0$ ) Manakov model like bright solitary wave solution can exist. With decreasing velocity the effective coupling constant $\kappa$ also decreases, which interestingly influences the intensity of the soliton to increase and reflects a possible nonintegrable property of the model. Finally for the soliton velocity $v=-\frac{1}{\rho_{0}}$ the nonlinear term to sustain the soliton disappears and hence no such soliton can appear anymore. However for the negative velocity below this value, $v<-\frac{1}{\rho_{0}}$, the sign of $\kappa$ flips and kink-like exact dark soliton can appear. For $\rho_{0}<0$ the whole picture reverts. This amazing solitonic feature evidently is a generalization of the chiral soliton property of [33] due to the presence of Manakov term as well as the multi-component nature and may have important applications in nonlinear optics. The suspected nonintegrable nature of this system and consequently the original chiral-solitonic system [33] can be convincingly proved by showing that the conserved quantities of the model are not in involution (in particular using the canonical bracket $(26)$ it can be shown that $\left\{c_{2}, c_{3}\right\} \neq 0$ ). Therefore though this system possesses Lax pair and infinite conserved quantities, their noninvolutiveness spoils the integrability. The involution of the conserved quantities however is restored if we use the noncanonical bracket (25) and this ensures the exact integrability of (9).

\section{CONCLUSION}

We have constructed the Lax pair of the proposed integrable CNLS equation (9) with cubic-quintic nonlinearity governing the soliton propagation in non-Kerr media, and using it generated the infinite set of its conserved quantities in explicit form. We also presented the exact one and two-soliton solutions of the model using those of the well known Manakov model. It has been demonstrated through the explicit two-soliton solution of the proposed model that the intensity of the t-derivative of soliton in the interaction region is much higher than that of the Manakov model. Moreover, the localized part of the time derivative of the Manakov soliton gets splitted and peaks around two values as shown in Figs 2 and 3. However, such splitting can be suppressed in the generalized cubic-quintic equation (9) having nonzero $\rho$ 's and $\tau$ 's as has been demonstrated in Figs. 4 and 5. These figures also confirm that the shape changing inelastic soliton collisions, as in the Manakov case, persist in our model. We believe that our results will be found equally useful in more general situations like Eq. (5) by taking our model as the unperturbed part and treating the remaining terms as perturbations.

We have also established the relationship between the proposed model and the Manakov model at the Lax pair level as well as at the field solution level, which shows an intriguing change in the canonical structure, namely the bosonic relations of Manakov model transforms into the anyonic relations of the present system. 
Another remarkable fact is that assuming the standard canonical structure for our fields we are able to derive from the same Hamiltonian yet another coupled system with cubic-quintic nonlinearity. This novel model, which turns out to be nonintegrable, represents a vector generalization of Aglietti et al model, famous for exhibiting chiral-soliton solutions. Such chiral-soliton property also prevails in the present vector case showing fascinating properties of the solitons, like changing intensity with soliton velocity, vanishing of bright solitons and appearance of dark solitons below certain velocity etc. Such properties may have important applications in nonlinear optical processes.

\section{ACKNOWLEDGEMENTS}

The work of R.R and M.L. forms part of a Department of Science and Technology research project.

[1] G.P.Agarwal, Nonlinear Fiber Optics - Second Edition (Academic Pres, New York, 1995).

[2] Yu. S. Kivshar and B. L. Davies, Phys. Rep. 298, 81 (1998).

[3] N. Akhmediev and A. Ankiewicz, Solitons: Nonlinear Pulses and Beams (Chapman \& Hall, London, 1997).

[4] H. A. Haus and W. S. Wong, Rev. Mod. Phys. 68, 423 (1996).

[5] A. Hasegawa and Y. Kodama, Solitons in Optical Communications (Oxford University Press, England, 1995).

[6] M. N. Islam, Ultrafast Fiber Switching Devices and Systems (Cambridge University Press, England, 1992).

[7] A. C. Newell and J. V. Moloney, Nonlinear Optics (Addison-Wesley, New York, 1992).

[8] F. Abdullaev, S. Darmanyan, P. Khabibullaev, Optical Solitons (Springer-Verlag, Berlin, 1993).

[9] A. E. Kaplan, Phys. Rev. Lett. 55, 1291 (1985); R. H. Enns, S. S. Rangenekar and A. E. Kaplan, Phys. Rev. A36, 1270 (1987); R. H. Enns, D. E. Edmundson, Phys. Rev. A47, 4254 (1993); A. Kumar, T. Kurz and W. Lauterbon, Phys. Lett A235, 367 (1997).

[10] D. I. Pushkarov and S. Tanev, Opt. Commun. 124, 354 (1996): S. Tanev and D. I. Pushkarov, Opt. Commun. 141, 322 (1997).

[11] A. Kumar and A. Kumar, Opt. Commun. 125, 377 (1996).

[12] J.M. Soto-Crespo and L. Pesquera, Phys. Rev. E56, 7288 (1997).

[13] C. Zhou, X. T. He and S. Chen, Phys. Rev. A46, 2277 (1992).

[14] V. V. Afanasjev, P. L. Chu and Yu. S. Kivshar, Opt. Lett. 22, 1388 (1997).

[15] V. Skarka, V. I. Berezhiani and R. Miklaszewski, Phys. Rev. E56, 1080 (1997).

[16] D. Artigas, L. Torner, J. P. Torres and N. Akhmediev, Opt. Commun. 143, 322 (1997); P. Honzatko, Opt. Commun. 127, 363 (1996).

[17] G. Dattoli, F. P. Orisitto and A. Toree, Opt. Lett. 14, 456 (1989).

[18] A. W. Snyder and A. P. Sheppard, Opt. Lett. 18, 482 (1993).

[19] C. Pare and M. Florjanczyk, Phys. Rev. A41, 6287 (1990) and references therein.

[20] M. Hisakado, T. Iizuka and M. Wadati. J. Phys. Soc. Jpn, 63, 2887 (1994).

[21] M. Hisakado and M. Wadati, J. Phys. Soc. Jpn, 63, 3962 (1994); 64, 408 (1995).

[22] S. V. Manakov, Zh. Eksp. Theor. Fiz. 65, 505 (1973) [Sov. Phys. JETP 38, 248 (1974)].

[23] R. Radhakrishnan, M. Lakshmanan and J. Hietarinta, Phys. Rev. E56, 2214 (1997).

[24] A. Hasegawa and T. Nyu, J. Lightwave Technol. LT11, 395 (1993).

[25] M. H. Jakubowski, K. Steiglitz and R. Squiev, Phys. Rev. E58, 6752 (1998).

[26] A. Ankiewicz and N. Akhmediev, Opt. Commun. 124, 95 (1996).

[27] S. Wabnitz, S. Trillo, E. M. Wright and G. I. Stegeman, J. Opt. Soc. Am. B8, 602 (1991); S. Trillo, S. Wabnitz, W. C. Banyai, N. Finlayson, C. T. Seaton, G. I. Stegeman and R. H. Stolen, IEEE. J. Quantum Electron QE25, 104 (1989).

[28] R. Radhakrishnan and M. Lakshmanan, Phys. Rev. E54, 2949 (1996).

[29] A. Kundu, J. Math. Phys, 25, 3433 (1984) ; F. Calogero, Inverse Problem, 3, 229 (1987) ; Li Shen in Symmetries E6 singularity structures (Springer, Ed. M. Lakshmanan, 1990), 27

[30] S. Kakei, N. Sasa and J. Satsuma, J. Phys. Soc. Jpn, 64, 1519 (1995).

[31] K. Kondo, K. Kajiwara and K. Matsui, J. Phys. Soc. Jpn, 66, 60 (1997).

[32] V. I. Zakharov and L. A. Takhtajan, Teor. Mat. Fiz. 38, 26 (1979); M. Lakshmanan (Ed.), Solitons: Introduction and Applications (Springer- Verlag, Berlin, 1988), p. 86.

[33] U. Aglietti, L. Griguolo, R. Jackiw, S.Y. Pi and D. Seminara, Phys. Rev. Lett. 77, 4406 (1996)

[34] A. Kundu, e-print hep-th/9811247 Exact solution of double- $\delta$ function Bose gas through interacting anyon gas.

[35] D. J. Kaup and B. A. Malomed, Phys. Rev. A48, 599 (1993); M. Karlsson, D. J. Kaup and B. A. Malomed, Phys. Rev. E54, 5802 (1996). 
[36] R. Radhakrishnan and M. Lakshmanan J. Phys. A: Math. Gen 28, 2683 (1995).

\section{FIGURES}
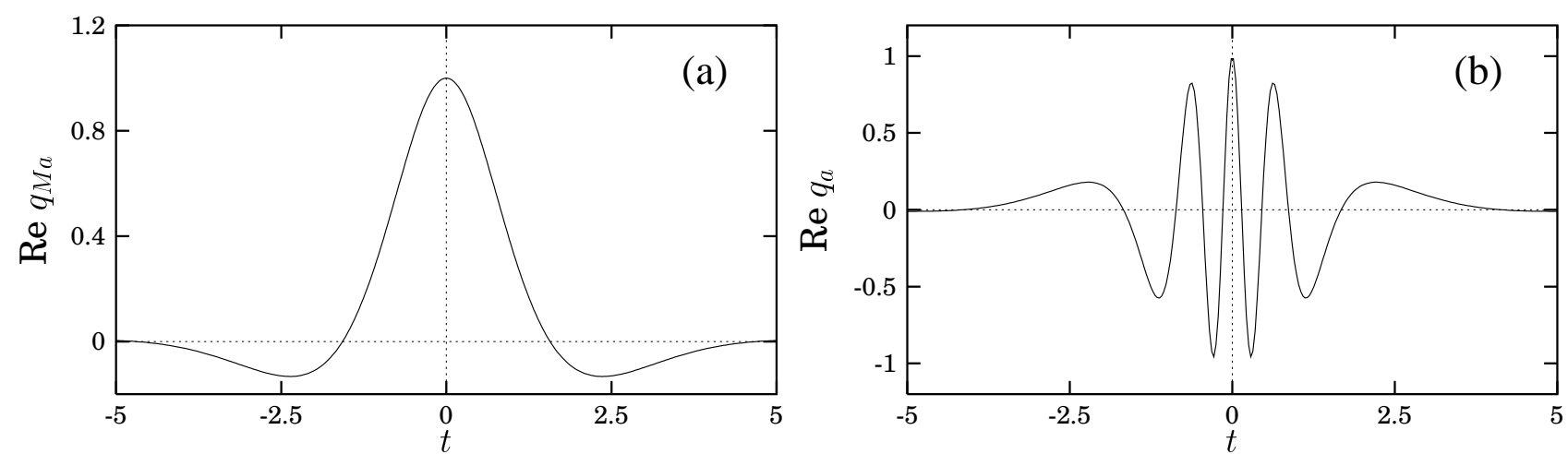

FIG. 1. Real part of the 1-soliton solution of (a) the Manakov model, (b) the quintic generalized Manakov model (see Eqn. (18)).
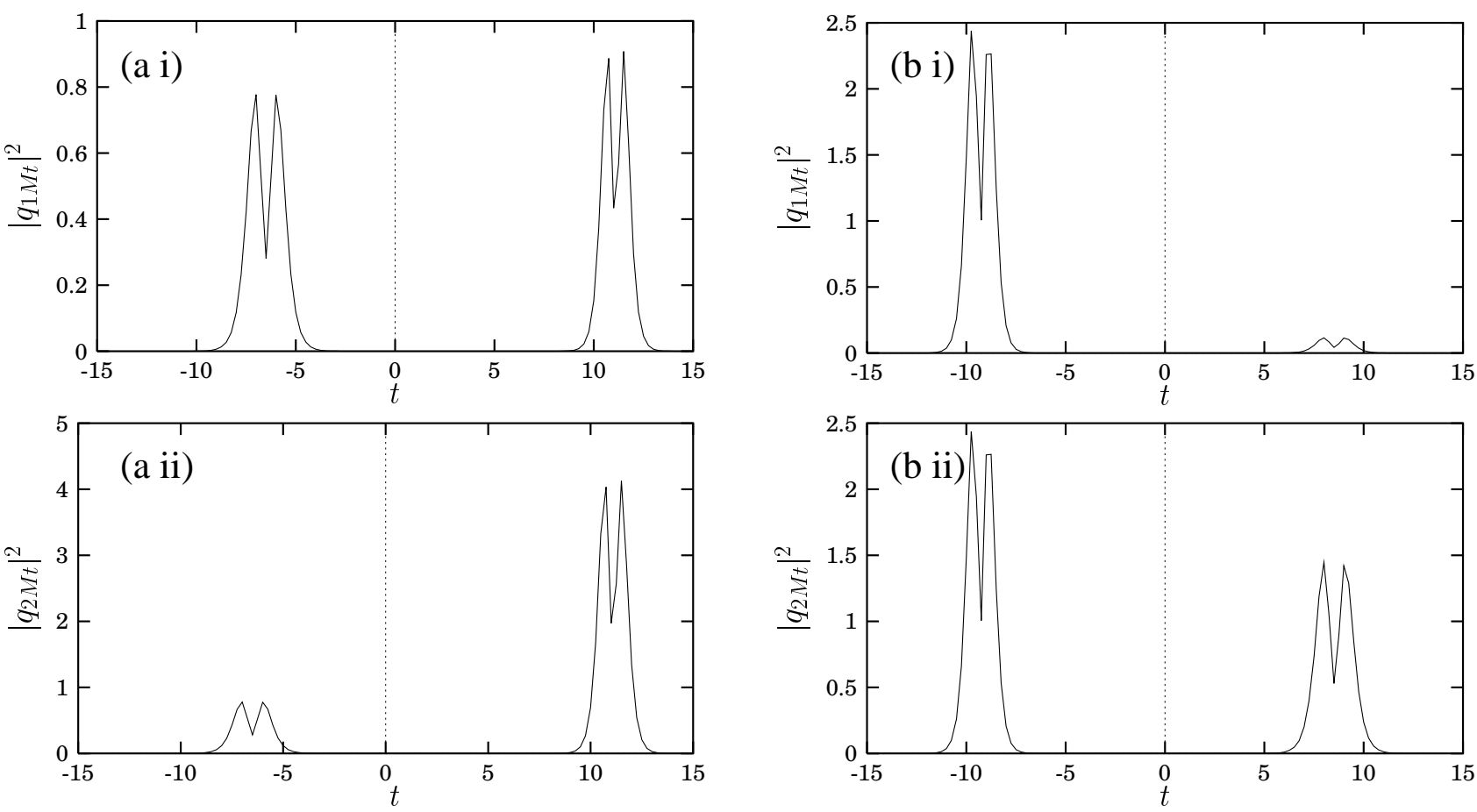

FIG. 2. Asymptotic forms of the intensity profiles $\left|q_{1 M t}\right|^{2}$ and $\left|q_{2 M t}\right|^{2}$ of the two-soliton solution (20) of the Manakov model $\left(\rho_{1}=\rho_{2}=\tau_{1}=\tau_{2}=0\right)$ with the parameter values $k_{1}=1.5+i 0.5, k_{2}=2.0-i 0.7, \alpha_{1}=\beta_{1}=\beta_{2}=1, \alpha_{2}=\frac{(39+i 80)}{89},(\mathrm{a})$ at $z=-7$ (before interaction), (b) at $z=+7$ (after interaction). Note the splitting in the asymptotic soliton profiles. 

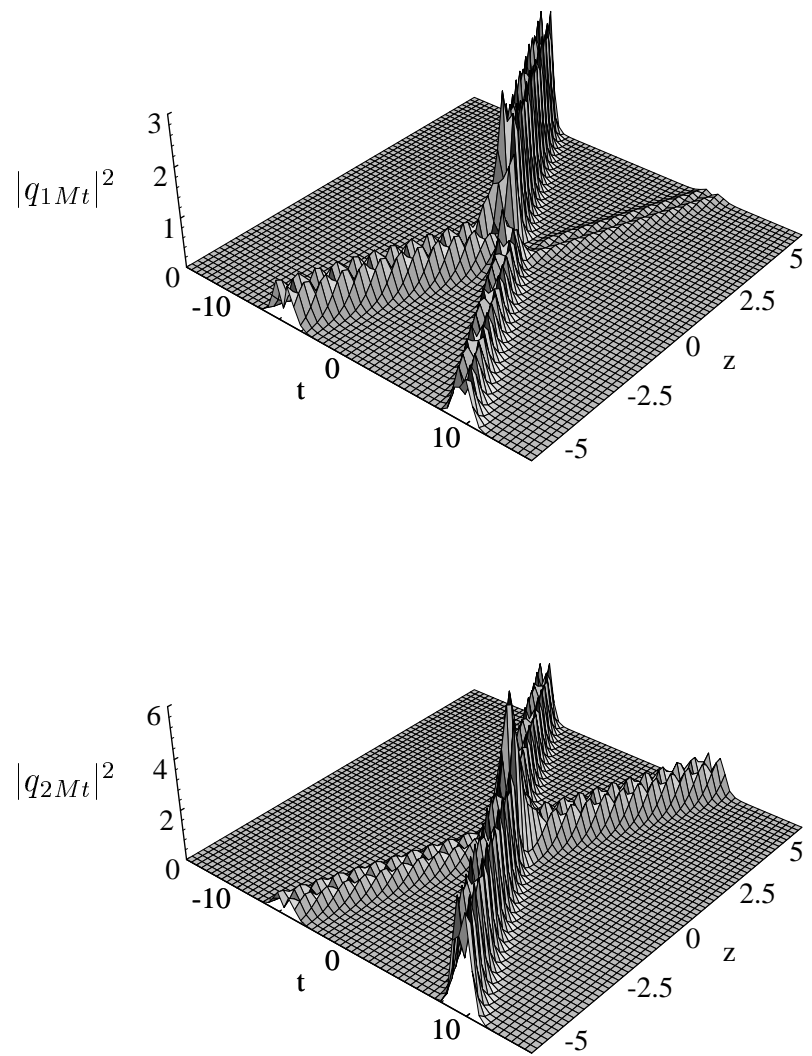

FIG. 3. Intensity profiles $\left|q_{1 M t}\right|^{2}$ and $\left|q_{2 M t}\right|^{2}$ of the two-soliton solution of the Manakov model with the parameteric values as in Fig. 目 

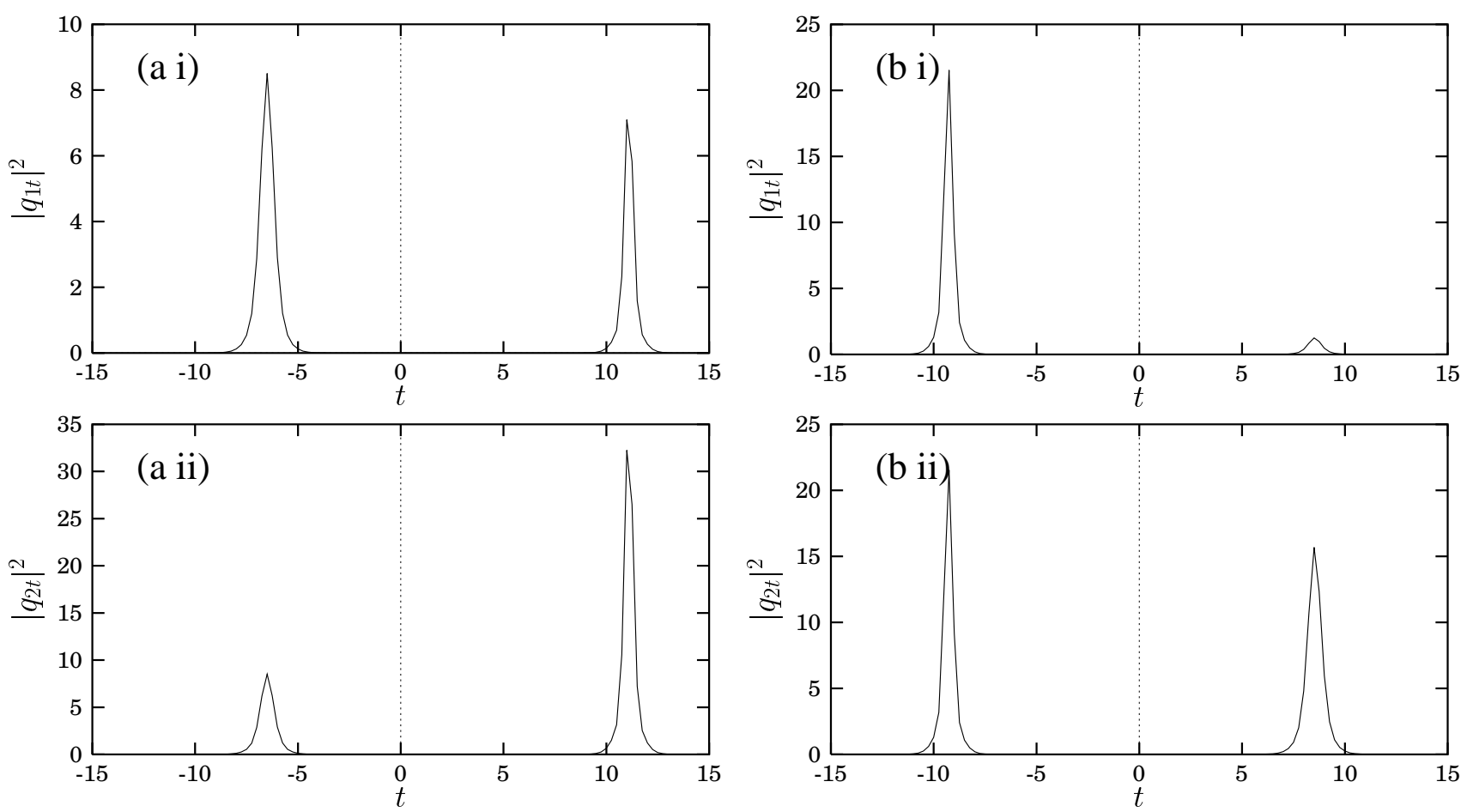

FIG. 4. Asymptotic forms of the intensity profiles $\left|q_{1 t}\right|^{2}$ and $\left|q_{2 t}\right|^{2}$ of the two-soliton solution (19) of the generalized model (9), (a) at $z=-7$, (b) $z=+7$, for nonzero values of the parameters $\rho_{1}=\rho_{2}=\tau_{1}=\tau_{2}=1$ and with remaining parameters as in Fig. 3. Note the suppression of the soliton splitting, which appeared in the asymptotic profile in Figs. 2 and 3 . 

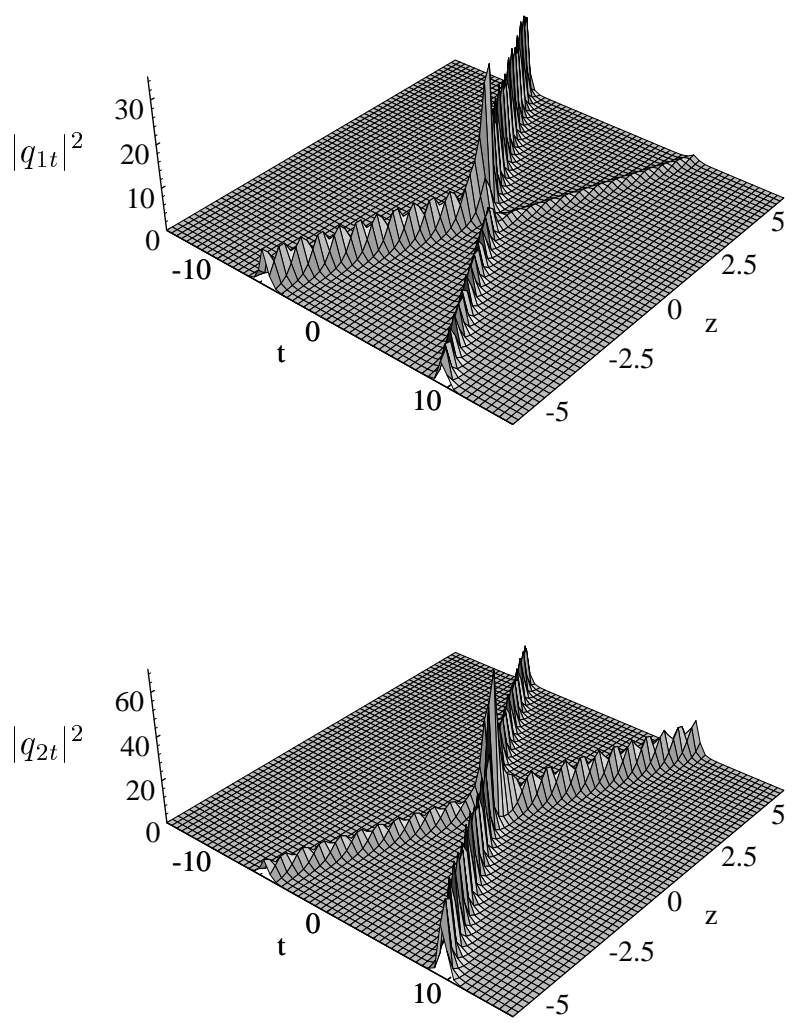

FIG. 5. Intensity profile $\left|q_{1 t}\right|^{2}$ and $\left|q_{2 t}\right|^{2}$ of the two-soliton solution (19) of the generalied Manakov model with the parameter values as in Fig. 4. Note the persistence of inelastic soliton collision as in the Manakov model and a higher intensity of modes during soliton interaction compared to the Manakov model. 